FORUM EMPRESARIAL

Vol. 18. Núm. 1 • verano 2013

\title{
Inversión empresarial en investigación y desarrollo (R\&D): iestrategia empresarial para el siglo XXI en Puerto Rico?
}

Manuel Lobato Vico / manuel.lobato@upr.edu

Universidad de Puerto Rico, Recinto de Río Piedras

Recibido: 5 de junio de 2012

Aceptado: 15 de febrero de 2013

RESUMEN:

La inversión de las empresas representa el $67 \%$ del total de recursos destinados a actividades de investigación y desarrollo (R\&D) en Puerto Rico. Partiendo del análisis de los resultados de la Encuesta Piloto sobre Investigación y Desarrollo, este trabajo concluye que en Puerto Rico hay un grupo de empresas de reciente creación muy enfocadas en actividades de R\&D, pero no se puede hablar de una estrategia generalizada ni de un impacto profundo. La inversión corporativa en R\&D en Puerto Rico está muy asociada con filiales de empresas de Estados Unidos y subsectores industriales concretos (en especial la industria química (farmacéutica) y servicios científicos y desarrollo).

Palabras clave: investigación y desarrollo (R\&D), estrategias empresariales, Puerto Rico, inversión privada

\section{ABSTRACT:}

Two thirds (67\%) of total investment in Research and Development (R\&D) activities in Puerto Rico is made by the business sector. This paper analyzes the data from the Pilot Survey on Research and Development and finds that a group of startups or new enterprises focused on R\&D activities has been developed, but the total amount of $R \& D$ investment is not enough to consider this as a generalized strategy or one of deep impact on the economy. Corporate investment in R\&D in Puerto Rico is strongly associated with subsidiaries of multinational corporations from the United States, as well as with specific industrial subsectors, in particular with the chemical industry (pharmaceutical) and the subsector of research and development services.

Keywords: research and development, R\&D, business strategy, Puerto Rico, corporate investment 


\section{RELEVANCIA DE LAS ACTIVIDADES DE INVESTIGACIÓN Y DESARROLLO}

Debido a su impacto sobre la productividad y la competitividad, la innovación es sin lugar a dudas uno de los elementos más mencionados de las estrategias empresariales contemporáneas, especialmente en los sectores de mayor intensidad en el uso de tecnologías (Bogliacino y Pianta, 2010; Christensen, 1997; Kumbhakar et al., 2010; Ortega-Argilés et al., 2009; RICYT, 2001; Voigt y Moncada-PaternóCastelló, 2009; Ziqi y Greenfield, 2000). Desde una óptica schumpeteriana se plantea que los procesos de innovación son parte esencial de toda corporación irrespectivamente de su tamaño o complejidad.

La innovación es un concepto muy amplio, que abarca desde la capacidad de una empresa para desarrollar nuevos productos o diseñar maneras ingeniosas de comercializarlos hasta la adopción de métodos de producción distintos o de formas novedosas de organización internas (Red Iberoamericana de Indicadores de Ciencia y Tecnología, RICYT, 2001). La innovación a veces es resultado de esfuerzos deliberados de equipos de trabajo constituidos con ese propósito, mientras que en otras ocasiones es un resultado no esperado de los procesos de producción o comercialización, fruto de la casualidad o encontrado en el camino del cumplimiento de otro propósito. Dentro del amplio espectro de actividades de innovación resulta especialmente interesante el estudio de lo que se conoce como la investigación y desarrollo (research and development, en inglés, o por sus siglas, R\&D). Este concepto aglutina las actividades creativas que realiza una entidad de manera sistemática para "incrementar el volumen de conocimientos" (Organización para la Cooperación y Desarrollo Económicos, OCDE, 2002: 30). Es un esfuerzo deliberado y sistemático que tiene por esencia la creatividad, y que debe contener un elemento apreciable de novedad o busca resolver incertidumbres científicas o tecnológicas. En la práctica, bajo el concepto de investigación y desarrollo se aglutinan actividades de investigación básica, que son trabajos orientados a desarrollar los fundamentos del conocimiento, sobre todo a nivel teórico; actividades de investigación aplicada, que son más dirigidas a objetivos prácticos específicos; y actividades de desarrollo experimental, que se enfocan directamente 
en la producción de nuevos materiales, productos o dispositivos, así como en nuevos procesos, sistemas o servicios (OCDE, 2002).

$\mathrm{Al}$ considerar las actividades de $\mathrm{R} \& \mathrm{D}$ estamos midiendo los recursos que las empresas destinan de manera deliberada y sistemática a desarrollar productos o servicios que constituyan en esencia una novedad en los mercados. Por lo tanto, evaluar la dimensión de estas actividades en el sector empresarial de una economía supone en buena medida comprender hasta qué punto la innovación es central en sus estrategias, aunque esta no queda acotada por el concepto. En cierta forma, la detección de actividades de R\&D en un sector puede ser un indicador de la presencia de la innovación como guía y directriz en muchos otros ámbitos, o de que la búsqueda de la innovación se ha convertido en un elemento medular en estas empresas.

Una vez aclarada la importancia del concepto, cabe preguntarse ¿hasta qué punto es relevante la actividad de R\&D en las empresas que operan en Puerto Rico? ¿Cuáles son los perfiles de las empresas que están activas en R\&D en Puerto Rico? ¿Pueden las actividades de $\mathrm{R} \& \mathrm{D}$ detectadas reflejar un cambio de estrategia en estas empresas para ser más competitivas en los mercados del siglo XXI?

Este trabajo utiliza como principal referencia los resultados de la Encuesta Piloto de Ciencia y Tecnología 2009: Investigación y Desarrollo (R\&D), llevada a cabo por el Instituto de Estadísticas de Puerto Rico y publicada en 2011. El informe final de la Encuesta ofrece información detallada y estimados sobre la actividad de R\&D en el sector empresarial y otros sectores utilizando la misma metodología que se usa a nivel internacional ${ }^{1}$. El uso de esta metodología permite la comparación de los datos sobre Puerto Rico con los de otros países.

Dentro del sector empresarial la Encuesta se enfocó en tres estratos:

- Empresas de más de 10 empleados con potencial de realizar actividades de R\&D. Se identificaron las empresas adscritas a

\footnotetext{
${ }^{1}$ Esta metodología, detallada en una publicación conocida como Manual de Frascati, ha sido definida en el marco de la OCDE y se revisa periódicamente.
} 
sectores industriales que en otros países se han asociado con frecuencia con actividades de $\mathrm{R} \& \mathrm{D}^{2}$. A estas se añadieron las empresas en incubadoras especializadas en ciencia y tecnología, empresas que a nivel mundial figuran como líderes en R\&D y cuya actividad en Puerto Rico no está limitada a la distribución de productos, y empresas promovidas por la Compañía de Fomento Industrial. Se contactó a un total de 588 empresas y se obtuvieron 360 respuestas $(61 \%)$. De las empresas que respondieron, 51 (14\%) indicaron realizar actividades de investigación y desarrollo.

- Empresas de menos de 10 empleados con potencial de realizar actividades de R\&D. Este estrato se abordó a través de una muestra probabilística. Se contactaron 208 empresas y se obtuvieron 136 respuestas (65\%). De éstas, 10 (7\%) indicaron que realizaban actividades de $\mathrm{R} \& \mathrm{D}$.

\footnotetext{
${ }^{2}$ Se analizaron los resultados de las encuestas de Estados Unidos y la Unión Europea. Los subsectores seleccionados fueron: Fabricación de productos farmacéuticos (NAICS 3254), Fabricación de computadoras y equipo periférico (3341), Fabricación de equipo de comunicación (3342), Fabricación de componentes electrónicos (3344), Fabricación de instrumentos de navegación, medición, médicos y de control (3345), Fabricación de automóviles y camiones (3361), Fabricación de carrocerías y remolques (3362), Fabricación de partes para vehículos automotores (3363), Fabricación de equipo aeroespacial (3364), Fabricación de equipo y material médico, dental y para laboratorio (3391), Servicios de arquitectura, ingeniería y actividades relacionadas (5413), Servicios de consultoría en computación (5415), Servicios de investigación científica y desarrollo (5417). Tienen también una intensidad de actividades de R\&D elevada (aunque en menor medida que los sectores anteriores) y se tomaron en cuenta también para el diseño de la muestra los siguientes sectores: Fabricación de productos químicos básicos (3251), Fabricación de hules, resinas y fibras químicas (3252) y otros subsectores de la industria química (325), Fabricación de maquinaria y equipo (333), otros subsectores de fabricación de equipo de computadoras, comunicaciones y otros electrónicos (334), Fabricación de equipo de generación eléctrica y aparatos y accesorios eléctricos (335), otros subsectores de fabricación de equipo de transporte (336), el subsector 339: Otras industrias manufactureras; edición de periódicos, revistas, libros y similares, excepto a través de internet (511), Otras telecomunicaciones (517), Proveedores de acceso a Internet, servicios de búsqueda en la red y servicios de procesamiento de información (518) y otros servicios profesionales, científicos y técnicos (54).
} 
- Empresas de más de 10 empleados en los demás sectores. Al igual que en el caso anterior, se suministró la encuesta a una muestra probabilística de las empresas en este estrato. Se contactaron 322 empresas, de las cuales 169 respondieron (52\%). En este estrato no se identificó ninguna empresa activa en investigación y desarrollo. Este resultado valida la decisión del equipo de investigador de concentrar la encuesta en los dos estratos anteriores, ya que la encuesta en este tercer estrato tenía como objetivo verificar si en Puerto Rico se desarrollan actividades de R\&D en sectores adicionales a los incluidos en los dos primeros estratos.

A partir de los resultados obtenidos en los cuestionarios de las empresas que llevan a cabo actividades de R\&D se realizaron los estimados correspondientes para cada subsector y para el conjunto de la economía.

Además de analizar los datos publicados en el informe de la Encuesta Piloto, para este trabajo se llevó a cabo una investigación sobre los datos originales obtenidos en la referida encuesta, con el propósito de profundizar sobre el perfil de las empresas y sus perspectivas de futuro en relación a su inversión en $R \& D^{3}$.

\section{Importancia de LA ACtividad de R\&D en Puerto Rico y PAPEL DE LAS EMPRESAS}

Según los estimados del Instituto de Estadísticas (2011), los recursos destinados a R\&D en Puerto Rico ascienden a $\$ 467$ millones, de los cuales $\$ 314$ millones (67\%) corresponden a inversiones de las empresas. En relación al Producto Interno Bruto (PIB), la actividad de $R \& D$ equivale al $0.49 \%$, y la inversión de las empresas al $0.33 \%$.

\footnotetext{
${ }^{3}$ Agradecemos al Instituto de Estadísticas de Puerto Rico su autorización para utilizar los datos originales que recopilamos para profundizar en las áreas objeto de análisis en este trabajo. Cabe señalar que el autor de este artículo fue el director de la Encuesta Piloto de Ciencia y Tecnología, 2009: Investigación y Desarrollo.
} 
El sector empresarial se sitúa por tanto como el principal protagonista de la actividad de investigación y desarrollo en Puerto Rico. Esto es cónsono con lo que ocurre en los países líderes en recursos destinados a investigación y desarrollo a nivel mundial, como los países escandinavos, Japón, Alemania y Estados Unidos (European Commission, 2006; European Commission, 2009; IEPR, 2011; National Science Foundation, 2008; Tübke y Hernández, 2011; Wolfe, 2007, 2008 y 2009). Sin embargo, a diferencia de estos países, principalmente de los países europeos, en Puerto Rico no se observa una actividad paralela de R\&D de gran magnitud por parte de otros sectores, como las universidades y las agencias gubernamentales.

En el caso de Puerto Rico se podría argumentar que el protagonismo de las empresas en las actividades de investigación y desarrollo es resultado de la falta de inversiones cuantiosas por parte de otros sectores, más que de un dinamismo empresarial llamativo en este ámbito. La comparación de la magnitud de los recursos destinados a $\mathrm{R} \& \mathrm{D}$ en relación a la producción de cada país refuerza esta percepción. Frente al $0.33 \%$ del PIB que representa la inversión empresarial en R\&D en Puerto Rico, en Estados Unidos la inversión de las empresas es equivalente al 2.01\%; en Japón, al 2.68\%; y en los países europeos líderes en recursos destinados a R\&D, como Finlandia, Suecia, Dinamarca o Alemania, esta inversión representa entre $1.92 \%$ y $2.83 \%$ de su PIB (véase Tabla 1 ).

Llama la atención que en los países latinoamericanos con una inversión en R\&D relativa a su PIB parecida a la de Puerto Rico, las empresas tienen un papel más modesto, con una aportación en la inversión total en R\&D que oscila entre el 47\% de México y el 18\% de Uruguay (véase Tabla 1). 
INVERSIÓN EMPRESARIAL EN INVESTIGACIÓN Y DESARROLLO (R\&D)

Tabla 1

Inversión en R\&D en diferentes países e importancia relativa del sector empresarial

\begin{tabular}{|c|c|c|}
\hline & $\begin{array}{l}\text { Proporción de los } \\
\text { recursos destinados a } \\
\text { R\&D en relación al PIB }\end{array}$ & $\begin{array}{l}\text { Proporción de la } \\
\text { inversión empresarial } \\
\text { en relación al total de } \\
\text { inversión en R\&D }\end{array}$ \\
\hline Puerto Rico & 0.49 & 67 \\
\hline$\ldots$ & $\ldots$ & \\
\hline Israel & 4.86 & 79 \\
\hline Suecia & 3.75 & 71 \\
\hline Finlandia & 3.73 & 71 \\
\hline Japón & 3.42 & 78 \\
\hline Corea del Sur & 3.37 & 75 \\
\hline Suiza & 3.01 & 73 \\
\hline Estados Unidos & 2.77 & 73 \\
\hline Dinamarca & 2.72 & 67 \\
\hline Austria & 2.68 & 71 \\
\hline Islandia & 2.65 & 55 \\
\hline Alemania & 2.63 & 68 \\
\hline Sing apur & 2.51 & 72 \\
\hline$\ldots$ & $\ldots$ & \\
\hline Brasil & 1.10 & 40 \\
\hline Chile & 0.67 & 46 \\
\hline Uruguay & 0.64 & 18 \\
\hline Argentina & 0.52 & 27 \\
\hline Costa Rica & 0.39 & 30 \\
\hline México & 0.37 & 47 \\
\hline
\end{tabular}

Fuentes: Encuesta piloto de Ciencia y Tecnología 2009: Investigación y Desarrollo (R\&D), Instituto de Estadísticas de Puerto Rico, 2011; bases de datos del UNESCO Institute for Statistics y del Banco Mundial. Los datos corresponden al último año disponible del periodo 2004-2010.

\section{SUBSECTORES EMPRESARIALES MÁS INTENSIVOS EN INVESTIGACIÓN Y DESARROLLO}

En Puerto Rico, la inversión empresarial en R\&D está muy concentrada en la industria química, y específicamente en el sector farmacéutico, aunque también hay una actividad destacada en otros 
subsectores, como el de servicios profesionales, científicos y técnicos. En este último tienen especial relevancia los servicios de investigación científica y desarrollo, en particular la investigación en temas agrícolas, y los servicios de consultoría en computación (IEPR, 2011; véase Tabla 2).

Tabla 2

Subsectores en los que se concentra la inversión empresarial en R\&D (2009)

\begin{tabular}{|c|c|c|c|}
\hline Subsector & $\begin{array}{l}\text { Código } \\
\text { NAICS }\end{array}$ & $\begin{array}{l}\text { Estimado de } \\
\text { gasto en R\&D } \\
\text { del subsector } \\
\text { (millones de } \$ \text { ) }\end{array}$ & Por ciento \\
\hline Industria química & 325 & $\$ 184.5$ & 58.7 \\
\hline $\begin{array}{l}\text { Servicios profesionales, científicos y } \\
\text { técnicos }\end{array}$ & 541 & $\$ 62.2$ & 19.8 \\
\hline $\begin{array}{l}\text { Fabricación de equipo de generación } \\
\text { eléctrica y aparatos y accesorios } \\
\text { eléctricos ( } 335 \text { ); Otras industrias } \\
\text { manufactureras (339) }\end{array}$ & 335,339 & $\$ 51.7$ & 16.4 \\
\hline $\begin{array}{l}\text { Fabricación de equipo de } \\
\text { computación, comunicación, medición } \\
\text { y de otros equipos, componentes y } \\
\text { accesorios electrónicos }\end{array}$ & 334 & $\$ 2.9$ & 0.9 \\
\hline Otros sectores / no identificado & & $\$ 12.8$ & 4.1 \\
\hline TOTAL & & $\$ 314.2$ & \\
\hline
\end{tabular}

Fuente: Encuesta piloto de Ciencia y Tecnología 2009:

Investigación y Desarrollo (R\&D),

Instituto de Estadísticas de Puerto Rico, 2011.

La encuesta realizada por el Instituto de Estadísticas (IEPR, 2011) permitió identificar 25 subsectores empresariales ${ }^{4}$ a nivel de 4 dígitos en el North American Industrial Classification System 
(NAICS), con actividades de investigación y desarrollo. Antes de revisar el perfil de las empresas que realizan actividades de $\mathrm{R} \& \mathrm{D}$, analizamos la evolución reciente de los subsectores a los que pertenecen, y en especial la de los ocho subsectores de actividad en los que están las empresas de más de 100 empleados identificadas como activas en $\mathrm{R} \& \mathrm{D}$, que es donde se concentra la inversión. Estos sectores son los de fabricación de productos farmacéuticos; fabricación de instrumentos de navegación; medición, médicos y de control; fabricación de equipo de generación y distribución de energía eléctrica; fabricación de otra maquinaria y equipo para la industria en general; fabricación de equipo y material para uso médico, dental y para laboratorio; servicios de arquitectura, ingeniería y actividades relacionadas; fabricación de pinturas, recubrimientos, adhesivos y selladores; y servicios de investigación científica y desarrollo.

\footnotetext{
${ }^{4}$ Los subsectores identificados son: Fabricación de productos farmacéuticos (3254), Fabricación de equipo de generación y distribución de energía eléctrica (3353), Servicios de investigación científica y desarrollo (5417), Servicios de consultoría en computación (5415), Cultivo de hortalizas (1112), Servicios de arquitectura, ingeniería y actividades relacionadas (5413), Fabricación de equipo y material para uso médico, dental y para laboratorio (3391), Fabricación de instrumentos de navegación, medición, médicos y de control (3345), Fabricación de computadoras y equipo periférico (3341), Telefonía tradicional, telegrafía y otras telecomunicaciones alámbricas (5171), Artistas y técnicos independientes (7115), Fabricación de pinturas, recubrimientos, adhesivos y selladores (3255), Reparación y mantenimiento de maquinaria y equipo agropecuario, industrial, comercial y de servicios (8113), Fabricación de embarcaciones (3366), Fabricación de maquinaria y equipo para la industria metalmecánica (3335), Servicios de contabilidad, auditoría y servicios relacionados (5412), Fabricación de sistemas de aire acondicionado, calefacción y de refrigeración industrial y comercial (3334), Edición de periódicos, revistas, libros y similares, excepto a través de internet (5111), Fabricación de otra maquinaria y equipo para la industria en general (3339), Instalaciones y equipamiento en construcciones (2382), Fabricación de productos químicos básicos (3251), Minería de minerales no metálicos (2123), Fabricación de estructuras metálicas y productos de herrería (3323), Fabricación de carrocerías y remolques (3362), y Fabricación de otros equipos y accesorios eléctricos (3359).
} 
Según la información que provee el Negociado del Censo del Gobierno de Estados Unidos a través de la publicación County Business Patterns (CBP), la evolución de estos ocho subsectores a lo largo de los últimos cinco años (2005-2009, último año disponible) tiene varios rasgos en común. En primer lugar, es notable la disminución en el número de empresas de más de 100 empleados, y sobre todo el de más de 250 empleados. De hecho, sólo aumenta el número de empresas de mayor tamaño en uno de los ocho subsectores bajo estudio (el de fabricación de equipo y material para uso médico, dental y para laboratorio ${ }^{5}$ ). De forma inversa, en la mayoría de los subsectores se puede ver un incremento en el número de empresas de menos de 100 empleados, y sobre todo, de menos de 10 empleados. De nuevo, el sector de fabricación de equipo y material para uso médico, dental y laboratorio constituye la excepción. También encontramos una disminución en las empresas de menos de 100 empleados del subsector de fabricación de equipo de generación y distribución de energía eléctrica (véase Tabla 3).

La dinámica de reducción significativa en el número de establecimientos de mayor tamaño se observa a nivel de toda la economía de Puerto Rico, y es especialmente intensa en algunos sectores como la manufactura y, sobre todo, en servicios profesionales, científicos y técnicos. Es probable que el factor que incide más en esta dinámica sea la recesión económica que experimenta Puerto Rico desde el 2006 hasta finales del 2011.

Otro factor a considerar en el estudio de esta dinámica es la crisis financiera y económica internacional del 2007-09. La crisis parece haber incidido también en los establecimientos de menor tamaño en el conjunto de la economía, con una reducción del $2 \%$ de los establecimientos de menos de 100 empleados, y del 3\% en los de menos de 10 empleados, entre 2005 y 2009, según datos del CBP. En la manufactura esta reducción es aún más significativa, aunque en los sectores relacionados con servicios profesionales, científicos y técnicos se observa un aumento del $2 \%$.

\footnotetext{
${ }^{5}$ En el sector de fabricación de pinturas, recubrimientos, adhesivos y selladores aumenta el número de empresas de más de 100 empleados, pero no el de empresas de más de 250. 
Tabla 3

Evolución entre 2005 y 2009 de los subsectores más intensivos en R\&D

\begin{tabular}{|c|c|c|c|c|c|c|c|}
\hline \multirow[b]{2}{*}{ NAICS } & & \multirow[b]{2}{*}{$\begin{array}{l}\text { Número de } \\
\text { establecimientos, } \\
2009\end{array}$} & \multicolumn{5}{|c|}{ Cambios en el número de establecimientos entre 2005 y 2009} \\
\hline & & & Total & $\begin{array}{l}\text { Más de } \\
250 \\
\text { empleados }\end{array}$ & $\begin{array}{c}\text { Más de } \\
100 \\
\text { empleados }\end{array}$ & $\begin{array}{l}\text { Menos de } \\
100 \\
\text { empleados }\end{array}$ & $\begin{array}{l}\text { Menos de } \\
10 \\
\text { empleados }\end{array}$ \\
\hline & Todos los sectores, Puerto Rico & 45,549 & $-3 \%$ & $.9 \%$ & $-13 \%$ & $-2 \%$ & $-3 \%$ \\
\hline $31-39$ & Manufactura & 2,014 & $-7 \%$ & $-15 \%$ & $-15 \%$ & $-5 \%$ & $-5 \%$ \\
\hline \multirow[t]{2}{*}{54} & Servicios profesionales, dentíficos y técnicos & 4,299 & $2 \%$ & $-67 \%$ & $-33 \%$ & $2 \%$ & $2 \%$ \\
\hline & Sectores intensivos en actividades de R\&D & & & & & & \\
\hline 3255 & $\begin{array}{l}\text { Fabricación de pinturas, recubrimientos, } \\
\text { adhesivos y selladores }\end{array}$ & 23 & $15 \%$ & $-100 \%$ & $100 \%$ & $0 \%$ & $50 \%$ \\
\hline 3254 & Fabricación de productos farmacéuticos & 50 & $-17 \%$ & $-27 \%$ & $-24 \%$ & $0 \%$ & $11 \%$ \\
\hline 3345 & $\begin{array}{l}\text { Fabricación de instrumentos de navegación, } \\
\text { medición, médicos y de control }\end{array}$ & 14 & $-18 \%$ & $-80 \%$ & $-33 \%$ & $-9 \%$ & $0 \%$ \\
\hline 3339 & $\begin{array}{l}\text { Fabricación de otra maquinaria y equipo } \\
\text { para la industria en general }\end{array}$ & 8 & $0 \%$ & $-100 \%$ & $-67 \%$ & $40 \%$ & $25 \%$ \\
\hline 3353 & $\begin{array}{l}\text { Fabricación de equipo de generación y } \\
\text { distribución de energía eléctrica }\end{array}$ & 22 & $-21 \%$ & $-14 \%$ & $-25 \%$ & $-19 \%$ & $-25 \%$ \\
\hline 5417 & $\begin{array}{l}\text { Servicios de investigación cientificay } \\
\text { desarrollo }\end{array}$ & 52 & $13 \%$ & $-100 \%$ & $-33 \%$ & $16 \%$ & $25 \%$ \\
\hline 3391 & $\begin{array}{l}\text { Fabricación de equipo y material para uso } \\
\text { médico, dental y para laboratorio }\end{array}$ & 73 & $-10 \%$ & $45 \%$ & $18 \%$ & $-20 \%$ & $-19 \%$ \\
\hline 5413 & $\begin{array}{l}\text { Servicios de arquitectura, ingenieríay } \\
\text { actividades relacionadas }\end{array}$ & 925 & $13 \%$ & $-100 \%$ & $-45 \%$ & $14 \%$ & $15 \%$ \\
\hline
\end{tabular}

Fuente: County Business Patterns, Negociado del Censo de los Estados Unidos, 2005 a 2009.

La evolución en los últimos años en los sectores más intensivos en actividades de R\&D en Puerto Rico parece haber seguido en general un patrón similar al del conjunto de sectores de servicios profesionales, científicos y técnicos, que se caracteriza por una caída muy significativa en el número de establecimientos de mayor tamaño, así como un aumento, de magnitud variable, en el número de establecimientos de menor tamaño; no obstante, entre los sectores de manufactura más intensivos en actividades de R\&D el comportamiento es dispar. De hecho, el comportamiento conjunto de estos sectores de manufactura podría ser parecido al del agregado de los sectores de manufactura de la economía.

El hecho de que la mayor inversión en actividades de $\mathrm{R} \& \mathrm{D}$ por parte de empresas de más de 100 empleados se identifique en sectores en los que hay una reducción significativa en el número de establecimientos con más de 100 empleados puede ser sintomático 
de que las empresas están adoptando estrategias empresariales enfocadas en la R\&D para añadir valor a sus productos y servicios en un contexto de gran competencia internacional y/o de adversidad para los negocios, o que son precisamente las empresas que ya estaban orientadas a las actividades de R\&D las que logran sobrevivir en la crisis que ha atravesado Puerto Rico. En la medida que no se disponen de datos anteriores al 2009 sobre la inversión subsectorial en $\mathrm{R} \& \mathrm{D}$, no es posible determinar si efectivamente la inversión en $\mathrm{R} \& \mathrm{D}$ en estos subsectores ha crecido en los últimos años, como resultado del deterioro de la coyuntura económica.

No obstante, los resultados de la Encuesta Piloto (IEPR, 2011) arrojan un poco más de luz sobre este tema. Al preguntar a las empresas sobre su previsión de gastos en R\&D durante el 2010 y durante el periodo 2010-2012, las que informan con más frecuencia que esa inversión aumentará o se mantendrá en el mismo nivel son las empresas de más de 100 empleados en los sectores más intensivos en $\mathrm{R} \& \mathrm{D}^{6}$. De igual manera, aunque los datos provistos por las empresas para los años 2007 y 2008 presenta muchas limitaciones para el análisis, parece claro que entre 2007 y 2008 se produjo un aumento en la inversión de las empresas de los sectores más intensivos en actividades de R\&D (véase Tabla 4). En las empresas de otros sectores ese aumento parece menos significativo, aunque se prolonga hasta el 2009.

Por lo tanto, parece establecerse a través de los datos de la Encuesta Piloto que la estrategia de invertir en actividades de R\&D en las empresas de los subsectores más intensivos en R\&D se ha reforzado aún en el contexto de recesión severa de la economía de los últimos años, y se asume como área de desarrollo en el futuro, al menos

\footnotetext{
${ }^{6}$ Nos referimos a los siguientes sectores: Fabricación de productos farmacéuticos (3254), Fabricación de pinturas, recubrimientos, adhesivos y selladores (3255), Fabricación de otra maquinaria y equipo para la industria en general (3339), Fabricación de instrumentos de navegación, medición, médicos y de control (3345), Fabricación de equipo de generación y distribución de energía eléctrica (3353), Fabricación de equipo y material para uso médico, dental y para laboratorio (3391), Servicios de arquitectura, ingeniería y actividades relacionadas (5413), y Servicios de investigación científica y desarrollo (5417).
} 
INVERSIÓN EMPRESARIAL EN INVESTIGACIÓN Y DESARROLLO (R\&D)

Tabla 4

Evolución prevista en las inversiones en R\&D por parte de las empresas

\begin{tabular}{|c|c|c|c|c|}
\hline & Aumentarán & $\begin{array}{c}\text { Serán } \\
\text { similares }\end{array}$ & Disminuirán & $\begin{array}{c}\text { No sabe / } \\
\text { No contesta }\end{array}$ \\
\hline $\begin{array}{l}\text { Empresas en sectores más intensivos en R\&D, } \\
\text { con más de } 100 \text { empleados }\end{array}$ & $25 \%$ & $58 \%$ & $0 \%$ & $17 \%$ \\
\hline $\begin{array}{l}\text { Empresas en sectores más intensivos en R\&D, } \\
\text { con menos de } 100 \text { empleados }\end{array}$ & $22 \%$ & $28 \%$ & $22 \%$ & $28 \%$ \\
\hline Empresas en otros sectores & $24 \%$ & $43 \%$ & $19 \%$ & $14 \%$ \\
\hline \multicolumn{5}{|c|}{ ¿Y durante los próximos tres años (periodo 2010-2012)? } \\
\hline & Aumentarán & $\begin{array}{c}\text { Serán } \\
\text { similares }\end{array}$ & Disminuirán & $\begin{array}{c}\text { No sabe / } \\
\text { No contesta }\end{array}$ \\
\hline $\begin{array}{l}\text { Empresas en sectores más intensivos en R\&D, } \\
\text { con más de } 100 \text { empleados }\end{array}$ & $42 \%$ & $33 \%$ & $0 \%$ & $25 \%$ \\
\hline $\begin{array}{l}\text { Empresas en sectores más intensivos en R\&D, } \\
\text { con menos de } 100 \text { empleados }\end{array}$ & $33 \%$ & $22 \%$ & $6 \%$ & $39 \%$ \\
\hline Empresas en otros sectores & $38 \%$ & $24 \%$ & $5 \%$ & $33 \%$ \\
\hline
\end{tabular}

Fuente: Instituto de Estadísticas de Puerto Rico.

en las empresas de mayor tamaño. Este hallazgo contrasta con los que otros investigadores han observado en Estados Unidos y Europa, donde se observa una reducción en el crecimiento de la inversión empresarial en R\&D al iniciarse la crisis de 2008, y un crecimiento negativo de esta inversión en el 2009 (Cincera, Cozza, Tübke y Voigt, 2010; Moncada-Paternó-Castelló, Voigt y Vivarelli, 2011).

\section{Perfil de las empresas que realizan actividades de R\&D en Puerto Rico}

La Encuesta Piloto (IEPR, 2011) ofrece información detallada sobre el perfil de las empresas que invierten en actividades de R\&D en Puerto Rico. Como se mencionó anteriormente, esta información ha sido complementada a través de la investigación sobre los datos crudos de la encuesta.

Las grandes corporaciones de Estados Unidos realizan con frecuencia actividades de R\&D en sus filiales (Hedge y Hicks, 2008). 
Uno de los hallazgos más destacados de este estudio es precisamente la relevancia de la inversión que realizan las filiales de empresas de Estados Unidos en Puerto Rico, ya que se estima que éstas son responsables del $91 \%$ del total de fondos empresariales destinados a R\&D (véase Tabla 5).

Tabla 5

Inversión en R\&D de filiales de empresas de Estados Unidos en Puerto Rico (2009)

\begin{tabular}{lcccc} 
Tipo de empresas & $\begin{array}{c}\text { Estimado de } \\
\text { gasto en R\&D } \\
\text { (millones de } \$ \text { ) }\end{array}$ & Por ciento & $\begin{array}{c}\text { Estimado del } \\
\text { número de } \\
\text { empresas }\end{array}$ & Por ciento \\
\hline $\begin{array}{l}\text { Filiales de empresas de } \\
\text { Estados Unidos }\end{array}$ & $\$ 285.4$ & 90.8 & 26 & 24.6 \\
$\begin{array}{l}\text { Filiales de empresas de } \\
\text { otros países }\end{array}$ & $\$ 1.9$ & 0.6 & 3 & 3.3 \\
Empresas de Puerto Rico & $\$ 26.9$ & 8.6 & 77 & 72.1 \\
\hline TOTAL & $\$ \mathbf{3 1 4 . 2}$ & & &
\end{tabular}

Fuente: Encuesta piloto de Ciencia y Tecnología 2009: Investigación y Desarrollo (R\&D), Instituto de Estadísticas de Puerto Rico, 2011.

Este dato es complementado con la información provista por la National Science Foundation (NSF) sobre la inversión en investigación y desarrollo que realizan empresas de Estados Unidos en Puerto Rico (National Science Foundation, 2008) ${ }^{7}$. Según las cifras publicadas por NSF, se ha producido un crecimiento muy acelerado desde el 2004 (y al menos hasta el 2007, última fecha disponible) en las cantidades invertidas por estas empresas, lo que vendría a corroborar la conclusión del apartado anterior (véase Gráfica 1).

\footnotetext{
${ }^{7}$ Estos datos son recopilados a través de cuestionario similares a los utilizados por el Instituto de Estadísticas de Puerto Rico en su Encuesta Piloto, suministrados directamente a los responsables de las empresas en sus sedes en Estados Unidos. Debe notarse que los datos estimados por NSF difieren de los estimados por el Instituto de Estadísticas de Puerto Rico para estas empresas, ya que NSF ofrece un estimado de inversión empresarial significativamente más elevado.
} 


\section{Gráfica 1}

Evolución de la inversión en R\&D de filiales de empresas de Estados Unidos en Puerto Rico, según datos de National Science Foundation

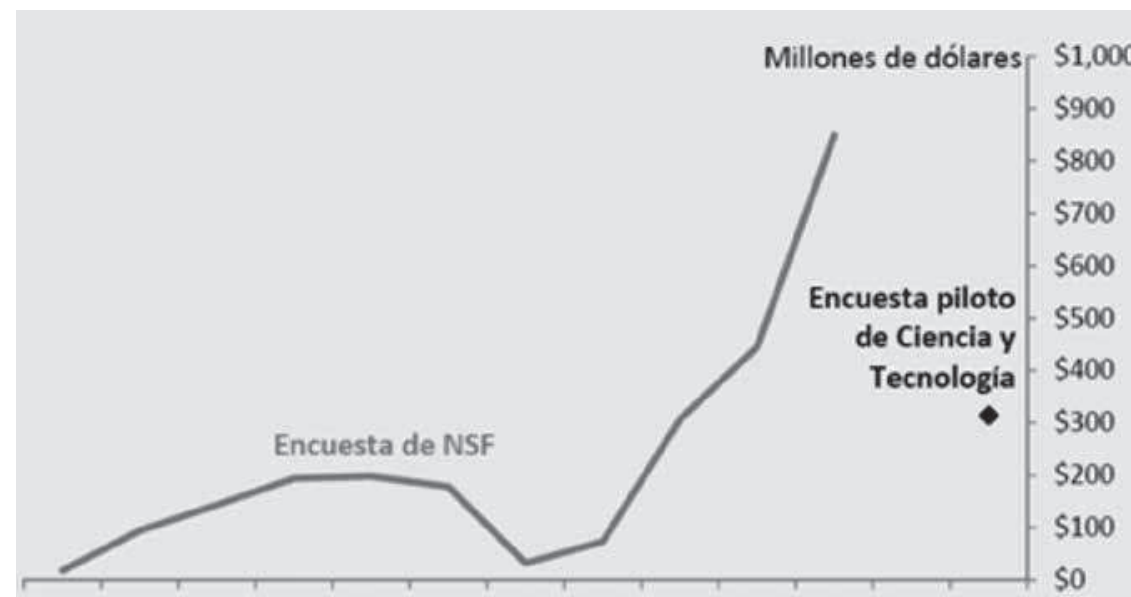

$19971998199920002001200220032004200520062007 \quad 20082009$

Fuente: National Science Foundation (NSF).

Gráfica reproducida del informe: Instituto de Estadísticas de Puerto Rico (2011) Encuesta Piloto de Ciencia y Tecnología, 2009: Investigación y Desarrollo, pág. 27.

La actividad de las filiales de empresas de Estados Unidos y de otros países (aunque en una cantidad mucho más reducida) que se observa en Puerto Rico es probablemente parte del fenómeno de "globalización de la R\&D corporativa". Las actividades de R\&D que eran consideradas antes actividades centrales y retenidas en las propias sedes de las grandes empresas, se desplazan ahora hacia otros países (Moncada-Paternó-Castelló, Voigt y Vivarelli, 2011). Trasciende el ámbito del trabajo que aquí se presenta el análisis detallado sobre los factores de atracción en el caso de Puerto Rico, dada la amplia diversidad de variables con las que se relacionan estas ubicaciones descentralizadas.

En cualquier caso, dada la importancia de las filiales de empresas de Estados Unidos en la inversión en R\&D en Puerto Rico, cabe preguntarse si el crecimiento observado y esperado en esta inversión 
se limita a una dinámica particular de las filiales de empresas de Estados Unidos en la isla. Los resultados de la Encuesta Piloto no parecen sostener esta idea. Aun con sus limitaciones, los datos que proveen las empresas encuestadas para los años 2007, 2008 y 2009 no señalan diferencias notables entre las dinámicas de las empresas filiales y las de empresas locales no filiales. En cuanto a la perspectiva hacia el futuro, las filiales de empresas de Estados Unidos encuestadas parecen más optimistas a corto plazo, aunque de nuevo, no encontramos diferencias significativas en sus proyecciones para el periodo 2010-2012 (véase Tabla 6).

Tabla 6

Evolución prevista en las inversiones en R\&D de empresas filiales y locales (2009)

¿Cuál es su previsión sobre los gastos en Investigación y Desarrollo dentro de su empresa en Puerto Rico durante el 2010?

\begin{tabular}{lcccc} 
& Aumentarán & $\begin{array}{c}\text { Serán } \\
\text { similares }\end{array}$ & Disminuirán & $\begin{array}{c}\text { No sabe / } \\
\text { No contesta }\end{array}$ \\
\cline { 2 - 5 } Empresas filiales & $30 \%$ & $55 \%$ & $10 \%$ & $5 \%$ \\
\hline Empresas locales no filiales & $23 \%$ & $38 \%$ & $23 \%$ & $15 \%$
\end{tabular}

¿Y durante los próximos tres años (periodo 2010-2012)?

\begin{tabular}{lcccc} 
& Aumentarán & $\begin{array}{c}\text { Serán } \\
\text { similares }\end{array}$ & Disminuirán & $\begin{array}{c}\text { No sabe / } \\
\text { No contesta }\end{array}$ \\
\cline { 2 - 5 } Empresas filiales & $36 \%$ & $32 \%$ & $0 \%$ & $32 \%$ \\
\hline Empresas locales no filiales & $37 \%$ & $20 \%$ & $7 \%$ & $37 \%$
\end{tabular}

Fuente: Instituto de Estadísticas de Puerto Rico.

El análisis por tamaño de las empresas activas en R\&D ofrece otras perspectivas interesantes, tanto en Puerto Rico como en otros países (Ortega-Argilés y Brandsma, 2009). Por un lado, la inversión en investigación y desarrollo se halla muy concentrada en las entidades de mayor tamaño, estimándose que el $63 \%$ proviene de empresas de 1,000 empleados o más, y un $11 \%$ adicional de empresas de 500 a 1,000 empleados en Puerto Rico (véase Tabla 7). Por otro lado, las 16 ISSN 1541-8561 FORUM EMPRESARIAL VOL. 18 NÚM. 1 •VERANO 2013 
INVERSIÓN EMPRESARIAL EN INVESTIGACIÓN Y DESARROLLO (R\&D)

empresas de menor tamaño juegan un papel muy relevante. Como indica el informe de la Encuesta Piloto, "dos de cada tres empresas involucradas en actividades de R\&D en Puerto Rico tienen menos de 50 empleados (68 por ciento), y en conjunto son responsables del 10\% de la inversión en R\&D” (IEPR, 2011: 26).

Tabla 7

Inversión empresarial en $\mathrm{R} \& \mathrm{D}$, por tamaño de la empresa (2009)

\begin{tabular}{lccc}
$\begin{array}{l}\text { Tamaño } \\
\text { (número de } \\
\text { empleados) }\end{array}$ & $\begin{array}{c}\text { Estimado de } \\
\text { gasto en R\&D } \\
\text { (millones de } \$ \text { ) }\end{array}$ & Por ciento & $\begin{array}{c}\text { Número de } \\
\text { empresas, } \\
\text { por ciento }\end{array}$ \\
\hline 1,000 o más & $\$ 191.5$ & 62.7 & $7 \%$ \\
500 a 999 & $\$ 34.6$ & 11.3 & $5 \%$ \\
250 a 499 & $\$ 15.5$ & 5.1 & $3 \%$ \\
100 a 249 & $\$ 29.5$ & 9.7 & $8 \%$ \\
50 a99 & $\$ 3.1$ & 1.0 & $8 \%$ \\
10 a 49 & $\$ 16.2$ & 5.3 & $38 \%$ \\
1 a9 & $\$ 13.8$ & 4.5 & $21 \%$ \\
0 & $\$ 1.3$ & 0.4 & $10 \%$ \\
No identificado & $\$ 8.7$ & & \\
\hline TOTAL & $\$ 314.2$ & &
\end{tabular}

Fuente: Encuesta piloto de Ciencia y Tecnología 2009: Investigación y Desarrollo (R\&D), Instituto de Estadísticas de Puerto Rico, 2011.

De igual manera, el informe de la Encuesta Piloto destaca, a partir de la comparación internacional, que el grupo de empresas de 50 a 500 empleados tiene "un potencial por desarrollar" (Instituto de Estadísticas, 2011: 26). Esta conclusión se basa en que en la mayoría de los países que publican datos detallados de la inversión empresarial en R\&D por tamaño de la empresa, la inversión de las empresas de 50 a 249 empleados, y de 250 a 500 empleados es relativamente mayor a la observada en Puerto Rico. Como se puede ver en la Tabla 8, la inversión empresarial en R\&D por empresas de estos 
tamaños en Puerto Rico equivale al $0.03 \%$ del PIB, mientras que en otros países asciende incluso al $0.26 \%$. De hecho, hay países con una inversión en R\&D en relación al PIB menor que Puerto Rico, que presentan cifras superiores de inversión en las empresas de 50 a 500 empleados.

Tabla 8

Comparación internacional de la inversión empresarial en $R \& D$, por tamaño de la empresa (2007)

\begin{tabular}{|c|c|c|c|c|c|c|c|c|}
\hline \multirow{3}{*}{ Pais } & \multirow{3}{*}{$\begin{array}{l}\text { Gasto en } \\
\text { R\&D en } \\
\text { relación al } \\
\text { PIB (2007) }\end{array}$} & \multicolumn{7}{|c|}{ Gasto en R\&D por tamaño de ka empresa, \% del PIB } \\
\hline & & & & núme & o de emple & ados & & \\
\hline & & 0 & 1 ag & 10 a 49 & 50 a 249 & 250 a 499 & 500 a999 & $\begin{array}{c}1000 \text { o } \\
\text { más }\end{array}$ \\
\hline Finlan dia & 2.20 & & 0.05 & 0.14 & 0.21 & 0.21 & & \\
\hline Corea del Sur & 2.20 & & 0.02 & 0.19 & 0.26 & 0.12 & 0.14 & 1.55 \\
\hline Suecia & 2.26 & & & 0.14 & 0.26 & 0.17 & 0.15 & 1.55 \\
\hline Estados Unidos & 1.72 & & & & 0.15 & 0.05 & 0.10 & 1.30 \\
\hline Alemania & 1.62 & & 0.01 & 0.04 & 0.12 & 0.09 & & \\
\hline Dinamarca & 1.53 & & 0.09 & 0.12 & 0.26 & 0.10 & & \\
\hline Francia & 1.03 & 0.00 & 0.01 & 0.06 & 0.12 & 0.08 & & \\
\hline Portugal & 0.56 & & 0.01 & 0.05 & 0.14 & 0.08 & 0.04 & 0.23 \\
\hline España & 0.53 & 0.00 & 0.02 & 0.11 & 0.15 & 0.06 & 0.05 & 0.14 \\
\hline Hungría & 0.36 & 0.00 & 0.01 & 0.03 & 0.03 & 0.04 & 0.04 & 0.20 \\
\hline Puerto Rico (2009) & 0.33 & 0.00 & 0.01 & 0.02 & 0.03 & 0.02 & 0.04 & 0.20 \\
\hline Croacia & 0.25 & & 0.00 & 0.02 & 0.07 & 0.16 & & \\
\hline Letonia & 0.18 & & 0.01 & 0.03 & 0.05 & 0.00 & & \\
\hline Bulgaria & 0.14 & 0.00 & 0.01 & 0.01 & 0.04 & 0.00 & 0.00 & 0.07 \\
\hline Polonia & 0.14 & 0.00 & 0.00 & 0.01 & 0.04 & 0.03 & 0.03 & 0.04 \\
\hline Eslovaquia & 0.13 & 0.00 & 0.00 & 0.01 & 0.05 & 0.01 & 0.03 & 0.04 \\
\hline Rumania & 0.11 & & 0.00 & 0.01 & 0.02 & 0.02 & 0.02 & 0.05 \\
\hline Chipre & 0.07 & 0.00 & 0.02 & 0.01 & 0.01 & 0.01 & 0.02 & 0.00 \\
\hline
\end{tabular}

Fuente: Encuesta piloto de Ciencia y Tecnología 2009: Investigación y Desarrollo (R\&D), Instituto de Estadísticas de Puerto Rico, 2011.

Por último, cabe destacar que el perfil de las empresas que invierten en R\&D en Puerto Rico es también heterogéneo si se analiza la importancia relativa que tiene esta inversión en el conjunto de

18 ISSN 1541-8561 FORUM EMPRESARIAL VOL. 18 NÚM. 1 •VERANO 2013 
actividades de la empresa. Mientras algunas empresas están principalmente orientadas a desarrollar actividades de R\&D, en otras estas actividades son de carácter marginal, aunque no por ello deja de ser relevante (Potters, 2009). La comparación de la inversión realizada con los ingresos totales (ventas de bienes y servicios y otros ingresos) permite ilustrar esta diferencia en la importancia relativa de la inversión. Aunque una elevada proporción de las empresas encuestadas en la Encuesta Piloto no provee datos suficientes para hacer este análisis, entre aquellas que sí suministran la información, el 12\% realizan inversiones equivalentes al $40 \%$ de sus ingresos o superiores, y un $26 \%$ adicional dedican entre el $10 \%$ y el $40 \%$. En el otro extremo, el $21 \%$ de las empresas que proveen los datos invierten cantidades que equivalen al 1\% de sus ingresos o menos (véase Tabla 9).

Tabla 9

Importancia relativa de la inversión de las empresas en R\&D (2009)

\begin{tabular}{lcc}
$\begin{array}{c}\text { Inversión en R\&D / Ingresos } \\
\text { totales de la empresa }\end{array}$ & $\begin{array}{c}\text { Por ciento de } \\
\text { empresas } \\
\text { encuestadas }\end{array}$ & $\begin{array}{c}\text { Por ciento, entre } \\
\text { empresas que lo } \\
\text { especifican }\end{array}$ \\
\hline No especificado & $46 \%$ & \\
Entre $40 \%$ y $100 \%$ & $6 \%$ & $12 \%$ \\
Entre $10 \%$ y $40 \%$ & $14 \%$ & $26 \%$ \\
Entre $5 \%$ y $10 \%$ & $8 \%$ & $15 \%$ \\
Entre $1 \%$ y $5 \%$ & $14 \%$ & $26 \%$ \\
Entre $0 \%$ y $1 \%$ & $11 \%$ & $21 \%$
\end{tabular}

Fuente: Instituto de Estadísticas de Puerto Rico.

Las empresas en las que las actividades de R\&D tienen más importancia relativa son principalmente empresas de servicios científicos, profesionales y técnicos, mientras aquellas en las que estas actividades representan una proporción menor de sus ingresos pertenecen casi siempre a sectores de manufactura. No obstante, se identificaron empresas manufactureras con una proporción de inversión en 
$\mathrm{R} \& \mathrm{D} /$ ingresos totales superior al $20 \%$, así como empresas de servicios científicos, profesionales y técnicos con una proporción menor de $1 \%$.

$\mathrm{Al}$ analizar la evolución reciente de la inversión en $\mathrm{R} \& \mathrm{D}$ de estas empresas no se encontró un patrón claro en relación a la importancia relativa de la inversión en comparación con su volumen de ingresos. En la perspectiva sobre el futuro a corto plazo, sin embargo, es más frecuente que las empresas con mayor importancia relativa de la inversión en R\&D contesten que anticipan un aumento de esta inversión, aunque los datos obtenidos no son suficientes para extraer conclusiones definitivas al respecto.

\section{¿ES LA INVERSIÓN EN INVESTIGACIÓN Y DESARROLLO UNA ESTRATEGIA EMPRESARIAL PARA EL SIGLO XXI?}

Con frecuencia se ha destacado la importancia de la investigación y la aplicación del conocimiento en el desarrollo económico (Bravo y García, 2011; Cimoli, 2005; Cimoli, Ferraz y Primi, 2007; Curi, 2010; Sampat, 2007). En Puerto Rico existen actualmente empresas totalmente enfocadas en el desarrollo de actividades de R\&D, y además existe un número significativo de empresas que realizan actividades de esta naturaleza aunque la cantidad de dinero invertida, tanto en términos totales como en comparación con sus ingresos, sea relativamente reducida.

La Encuesta Piloto (IEPR, 2011) sacó a relucir los detalles de esta inversión en $\mathrm{R} \& \mathrm{D}$ y recogió información sobre el perfil de las empresas que invierten. A partir de la información obtenida, ¿`se puede hablar de la inversión en R\&D como una nueva estrategia o iniciativa de las empresas para lograr mayor competitividad de los mercados en el siglo XXI?

Las empresas más concentradas en las actividades de $R \& D$ en Puerto Rico, es decir, las empresas en las que la inversión en $R \& D$ equivale al menos al $5 \%$ de su ingreso total, tenían una vida promedio de 12 años en el momento de ser encuestadas. Se crearon en torno a 1998, en promedio, y el desarrollo de sus actividades se ha dado en el marco del nuevo siglo. Las empresas con una inversión 
en $\mathrm{R} \& \mathrm{D}$ inferior al $5 \%$ de su ingreso total, por el contrario, tienen una vida promedio de 23 años (se crearon en torno a 1987, en promedio). Se podría hablar, por tanto, de que el concepto de empresas centradas en actividades de investigación y desarrollo germina en Puerto Rico fundamentalmente en los últimos diez o quince años, y que el interés en las actividades de $\mathrm{R} \& \mathrm{D}$ se está intensificando a pesar de, o quizás debido a, la crisis económica que atraviesa Puerto Rico desde el 2006.

Por otro lado, la inversión empresarial en R\&D en Puerto Rico está concentrada en subsectores específicos-industria química, servicios profesionales, científicos y técnicos, así como algunos otros subsectores de manufactura-y tiene como principales protagonistas a las filiales de empresas de Estados Unidos.

La comparación internacional muestra que la inversión en $\mathrm{R} \& \mathrm{D}$, tanto la inversión total como la empresarial, es significativamente mayor en Estados Unidos, los países de la Unión Europea o Japón. Las cifras de Puerto Rico son comparables con las de otros países de América Latina, como Argentina o México, aunque están por debajo de los niveles de Brasil. Desde esta perspectiva, no se puede hablar de que el mundo de los negocios en Puerto Rico haya incorporado de manera amplia la consideración de las actividades de R\&D en sus estrategias. Al analizar la inversión empresarial según los tamaños de las empresas, se pone de manifiesto que al menos las empresas de tamaño mediano (50 a 500 empleados) tienen que incrementar significativamente su inversión en R\&D para alinearse con los niveles que se observan a nivel internacional.

En conclusión, entendemos que sí se puede hablar de unas estrategias empresariales concentradas en actividades de $\mathrm{R} \& \mathrm{D}$ que son relativamente recientes, y que están presentes en sectores que han experimentado transformaciones profundas en las últimas décadas (en especial, el cierre de plantas o empresas). En ese sentido, la inversión en R\&D es una estrategia del siglo XXI en Puerto Rico. No obstante, debe señalarse que todavía no es una estrategia ampliamente extendida en la economía, como se ha mostrado en este trabajo a través del análisis de la magnitud de la inversión y del perfil de las empresas que dedican recursos a actividades de R\&D. 


\section{Referencias}

Bravo, C. y García, A. (2011). R\&D and productivity: A two way avenue. World Development, 39(7), 1090-1107.

Bogliacino, F. y Pianta, M. (2010). Profits, R\&D and Innovation: a model and a test. IPTS Working Paper on Corporate REDD and Innovation, 5. Recuperado de http://iri.jrc.ec.europa.eu/papers/2010_JRC58871_WP5.pdf

Christensen, C. (1997). The innovator's dilemma: When new technologies cause great firms to fail. Cambridge, MA: Harvard Business School Press.

Cimoli, M. (ed.). (2005). Heterogeneidad estructural, asimetrías tecnológicas y crecimiento en América Latina. Chile: Naciones Unidas. Recuperado de http://www.eclac.cl/iyd/noticias/paginas/4/31434/ W35_CIMOLI.pdf

Cimoli, M., Ferraz, J. C. y Primi, A. (2007). Políticas de ciencia y tecnología en economías abiertas: la situación de América Latina y el Caribe. Serie Desarrollo Productivo, 165. Chile: CEPAL, Naciones Unidas. Recuperado de http://www.eclac.cl/iyd/noticias/ paginas $/ 5 / 31425 /$ serie165esp.pdf

Cincera, M., Cozza, C., Tübke, A. y Voigt, P. (2010). Doing R\&D or not, that is the question (in a crisis...). IPTS Working Paper on Corporate REDD and Innovation, 12. Recuperado de http:/ /iri.jrc. ec.europa.eu/papers /2010_JRC62614_WP12.pdf

Curi, V. (2010). Economía del conocimiento: difusión e integración en Puerto Rico. Tesis de maestría, Departamento de Economía, Universidad de Puerto Rico, Recinto de Río Piedras.

European Commission. (2006). The Annual Digest of Industrial RED. Sevilla, Spain: Directorate General Joint Research Center, European Communities.

European Commission. (2009). The 2009 EU Industrial REDD Investment Scoreboard. Luxembourg: European Commission Joint Research Center, European Communities.

Hegde, D. y Hicks, D. (2008). The maturation of global corporate R\&D: Evidence from the activity of U.S. foreign subsidiaries. Research Policy, 37(3), 390-406. 
INVERSIÓN EMPRESARIAL EN INVESTIGACIÓN Y DESARROLLO (R\&D)

Instituto de Estadísticas de Puerto Rico. (2011). Encuesta Piloto de Ciencia y Tecnología, 2009: Investigación y Desarrollo (REDD). San Juan, Puerto Rico: Instituto de Estadísticas de Puerto Rico (IEPR). Recuperado de http://www.estadisticas.gobierno. $\mathrm{pr} /$ iepr $/$ LinkClick.aspx?fileticket=hm2vGmuwZ18\%\}3d\&tab $\mathrm{id}=165$

Kumbhakar, S., Ortega-Argilés, R., Potters, L., Vivarelli, M. y Voigt, P. (2010). Corporate R\&D and firm efficiency: Evidence from Europe's top R\&D investors. IPST Working Paper on Corporate $R \mathcal{E} D$ and Innovation, 11. Luxembourg: European Commission. Recuperado de http://iri.jrc.ec.europa.eu/papers/2010_ JRC60762_WP11.pdf

Moncada-Paternó-Castelló, P., Voigt, P. y Vivarelli, M. (2011). Evolution of globalised business R\&D - Features, drivers, impacts. IPST Working Paper on Corporate REDD and Innovation, 2-2011. Luxembourg: European Commission.

National Science Foundation. (2008). Research and development in industry: 2004. Division of Sciences Resources Statistics, National Science Foundation. [Se consultaron también los informes correspondientes a los años 1995-96, 1997, 1998, 1999, 2000, 2001 y 2002]. Recuperado de http://www.nsf.gov/statistics/industry/

Organización para la Cooperación y Desarrollo Económicos. (2002). Manual de Frascati: propuesta de norma práctica para encuestas de investigación y desarrollo experimental. Fundación Española de Ciencia y Tecnología (FECYT), por acuerdo con la Organización para la Cooperación y Desarrollo Económicos (OCDE).

Ortega-Argilés, R. y Brandsma, A. (2009). EU-US differences in the size of R\&D intensive firms. IPST Working Paper on Corporate $R E D D$ and Innovation, 2-2009. Luxembourg: European Commission. Recuperado de http://iri.jrc.ec.europa.eu/papers.htm

Ortega-Argilés, R., Piva, M., Potters, L. y Vivarelli, M. (2009). Is corporate R\&D investment in high tech sectors more effective? IPST Working Paper on Corporate REDD and Innovation, 9-2009. Luxembourg: European Commission. Recuperado de http:// iri.jrc.ec.europa.eu/papers.htm 
Ortega-Argilés, R., Potters, L. y Vivarelli, M. (2009). R\&D and productivity: Testing sectoral peculiarities using micro data. IPST Working Paper on Corporate REDD and Innovation, 3-2009. Luxembourg: European Commission. Recuperado de http://iri.jrc. ec.europa.eu/papers.htm

Potters, L. (2009). R\&D in low tech sectors. IPST Working Paper on Corporate REDD and Innovation, 8-2009. Luxembourg: European Commission. Recuperado de http://iri.jrc.ec.europa.eu/papers.htm

Red Iberoamericana de Indicadores de Ciencia y Tecnología. (2001). Manual de Bogotá: normalización de indicadores de innovación tecnológica en América Latina y el Caribe. Red Iberoamericana de Indicadores de Ciencia y Tecnología (RICYT), Organización de Estados Americanos (OEA) / Programa CYTED.

Sampat, B. (2007). Política científica y tecnológica de Estados Unidos: reseña histórica e implicancias para los países en desarrollo. Chile: Comisión Económica para América Latina y el Caribe (CEPAL), Naciones Unidas. Recuperado de http://www.eclac.cl/iyd/noticias/paginas/4/31424/sampat.pdf

Tübke, A. y Hernández, H. (2011). The 2011 EU survey on REDD investment business trends. Luxembourg: European Commission. Recuperado de http://ftp.jrc.es/EURdoc/JRC65992.pdf

UNESCO Institute for Statistics. (2003). Immediate, medium and longerterm strategy in science and technology statistics. Montreal: UNESCO Institute for Statistics.

UNESCO Institute for Statistics. (2009). A global perspective on research and development. UIS Fact Sheet, 2, October 2009. Montreal: UNESCO Institute for Statistics.

UNESCO Institute for Statistics. (2010). Measuring R\&D: Challenges faced by developing countries. Technical Paper 5. Montreal: UNESCO Institute for Statistics.

U.S. Census Bureau. (2009). County Business Patterns. U.S. Census Bureau, US Department of Commerce. Recuperado de http:/ / www.census.gov/econ/cbp/

Voigt, P. y Moncada-Paternó-Castello, P. (2009). The global economic and financial downturn: What does it imply for firms' R\&D 
INVERSIÓN EMPRESARIAL EN INVESTIGACIÓN Y DESARROLLO (R\&D)

strategies? IPST Working Paper on Corporate RED and Innovation, 12-2009. Luxembourg: European Commission. Recuperado de http://iri.jrc.ec.europa.eu/papers.htm

Wolfe, R. (2007). Expenditures for U.S. industrial R\&D continue to increase in 2005; R\&D performance geographically concentrated. InfoBrief, NSF 07-335, National Science Foundation. Recuperado de http:/ / www.nsf.gov/statistics/infbrief/nsf07335/

Wolfe, R. (2008) U.S. business R\&D expenditures increase in 2006; Companies' own and federal contributions rise. InfoBrief, NSF 08-313. National Science Foundation. Recuperado de http:// www.nsf.gov/statistics/infbrief/nsf08313/

Wolfe, R. (2009). U.S. business R\&D expenditures increase in 2007; Small companies performed $19 \%$ of nation's business R\&D. InfoBrief, NSF 09-316, National Science Foundation. Recuperado de http://www.nsf.gov/statistics/infbrief/nsf09316/

Ziqi, L. y Greenfield, P. (2000).The synergy of corporate R\&D and competitive strategies: An exploratory study in Australian hightechnology companies. The Journal of High Technology Management Research, 11(1), 93-107. 
